\title{
Hypoxia and an Angiogenic Response in the Partially Obstructed Rat Bladder
}

\author{
Mohamed A. Ghafar, Aristotelis G. Anastasiadis, L. Eric Olsson, Paul Chichester, \\ Steven A. Kaplan, Ralph Buttyan, and Robert M. Levin
}

The Department of Urology (MAG, AGA, LEO, SAK, RB) and The Department of Pathology (RB), The College of Physicians and Surgeons of Columbia University, New York; and The Albany College of Pharmacy (PC, RML), Stratton Veterans Administration Medical Center, Albany, New York

\begin{abstract}
SUMMARY: Previous molecular and blood flow studies performed on animal models of partial bladder outlet obstruction (PBOO) caused us to propose that bladder hypoxia/ischemia was a significant effector of the cellular and functional changes that occur in the bladder as a result of this condition. To confirm the occurrence of hypoxia in the partially obstructed bladder, we obtained rat bladders at increasing intervals following $\mathrm{PBOO}$ and measured biomarkers of hypoxia (intracellular formation of hypoxyprobe- 1 adducts and expression of hypoxia inducible factor- $1 \alpha$ [HIF-1 $\alpha$ ] protein) and whether such hypoxia might elicit an angiogenic response in the tissue. Rats receiving PBOO or controls were treated with hypoxyprobe- 1 at increasing intervals subsequent to surgery and their bladders were sectioned and immunostained using an antibody that detects hypoxyprobe-1 adducts. Control rat bladders were unstained, whereas intense, but regionally restricted, hypoxyprobe- 1 immunostaining was detected in all obstructed bladders in a unique pattern that changed over time. Proteins were extracted from bladders removed from similarly treated rats and were analyzed for the expression of the $\mathrm{HIF}-1 \alpha$ protein as well as for expression of angiogenic regulatory factors (vascular endothelial growth factor, angiopoietin-1, and endostatin) using Western blotting techniques. HIF-1 $\alpha$ protein was not expressed in control bladders, however, the protein was highly up-regulated over the 2-week period after PBOO. Likewise, the expression of vascular endothelial growth factor (a downstream target of HIF-1 $\alpha$ action) and angiopoietin- 1 was also up-regulated in obstructed bladders confirming an angiogenic response to this hypoxia. Enigmatically, however, expression of the antiangiogenic molecule endostatin was also up-regulated by chronic PBOO. These results further support the concept that hypoxia is involved in the cellular remodeling as well as in the progressive functional impairment exhibited by the urinary bladder after PBOO. (Lab Invest 2002, 82:903-909).
\end{abstract}

$P$ artial bladder outlet obstruction (PBOO), a common complication of benign prostatic hyperplasia, can lead to severe bladder dysfunctions in affected men (Wein, 1995). These dysfunctions can be associated with a drastic loss of smooth muscle function and bladder contractility in some cases, whereas other cases develop severely hypertrophied and hypercontractile bladders (Blaivas, 1996). This condition is mimicked in experimental animal models wherein a partial surgical ligation is placed around the urinary bladder outlet (of a rat or rabbit). The ligature restricts bladder output and can subsequently lead to the development of bladder dysfunctions in the animals that are similar to those observed in men with

\section{DOI: 10.1097/01.LAB.0000021135.87203.92}

Received February 28, 2002.

This work was supported by research grants from the Veterans' Administration and the National Institutes of Health (RO1-DK53965, -DK26508, and-DK47949). Partial support for M.A. Ghafar was provided through the Irwin White Fellowship for Prostate Cancer Research. Partial support for A.G. Anastasiadis was provided by the American Foundation for Urologic Disease (A.F.U.D.) Research Scholarship sponsored by AstraZeneca, Inc.

Address reprint requests to: Dr. Robert M. Levin, Director of Research, Albany College of Pharmacy, 106 New Scotland Avenue, Albany, NY 12208.E-mail:levinr@acp.edu benign prostatic hyperplasia (Levin et al, 1986, 1999). Results of research studies on these animal models have led us to propose that bladder outlet obstruction disrupts bladder blood flow and that the resulting ischemia/hypoxia of this tissue is the impetus for the initial bladder hypertrophy as well as the subsequent dysfunctions that develop in this tissue (Levin et al, 1986, 1999). Characterization of the effects of PBOO on the gene expression patterns of the rabbit bladder has demonstrated that they are similar to those that occur when the rabbit bladder is subject to partial ischemia (Buttyan et al, 1992). Moreover, direct measurement of blood flow in the rabbit PBOO model (using a microsphere-infusion method) has demonstrated a rapid early rise in bladder blood flow after PBOO (consistent with the period of hypertrophy) that is followed by a decline to levels significantly lower than normal in those bladders that develop contractile dysfunctions (Shabsigh et al, 2000).

The recent availability of a chemical probe for cellular hypoxia (hypoxyprobe-1 or pimonidazole) has enabled us to better re-evaluate the effects of PBOO on bladder cell oxygenation. This substance is a small molecular weight compound that freely diffuses through tissues but forms protein adducts in those cells, which are hypoxic (less than $10 \mathrm{~mm} \mathrm{Hg}$ at $37^{\circ} \mathrm{C}$ ). The protein-hypoxyprobe- 1 adducts can then 
be detected with simple immunohistochemical procedures using an antibody that recognizes them. Previously, this substance was used to demonstrate the onset of hypoxia in cultured cells and in tissues (in vivo) (Arteel et al, 1998). We have also taken advantage of our increased knowledge of the cellular response to hypoxia. Typically, hypoxia induces the expression of a $120 \mathrm{kd}$ protein referred to as hypoxia inducible factor- $1 \alpha$ (HIF-1 $\alpha$ ). HIF- $1 \alpha$ can dimerize with HIF-1 $\beta$ (also known as ARNT) to form a transcription factor that induces the downstream expression of gene products that better enable a cell to withstand the stress of hypoxia (glucose transport and glycolytic proteins) and, ultimately, compensate for the loss of oxygen by initiating an angiogenic response (Wang et al, 1995). In this study, we further evaluated the hypothesis that chronic PBOO is associated with bladder cell hypoxia in the rat PBOO model system and also asked whether such hypoxia might elicit an angiogenic response (indicated by the induction of vascular endothelial growth factor [VEGF] expression) in the bladder as an attempt to compensate for changes in tissue blood flow.

\section{Results}

Hypoxyprobe-1 Immunostaining in Partially Obstructed Rat Bladders

Control (unoperated), sham operated, or rats receiving PBOO were maintained for up to 2 weeks and groups $(n=4)$ were killed at 3 days or at 1 or 2 weeks after PBOO. These rats were injected with a solution of hypoxyprobe-1 2 hours before killing. Hypoxyprobe-I is a substituted 2-nitrominidazōe (pimonidazole hydrochloride) that is widely used to demonstrate hypoxic conditions in tissues (in vivo) and cultured cells ${ }^{7,9,10}$. Once injected into a laboratory animal or human, it is rapidly distributed to all tissues in the body but only forms adducts with proteins in cells having oxygen concentrations less than $14 \mu \mathrm{m}$, which is equivalent to a $\mathrm{pO}_{2}$ of $10 \mathrm{~mm} \mathrm{Hg}$ at $37^{\circ} \mathrm{C}$ (Shabsigh et al, 2000). Certain cells of the normal kidney are naturally reactive to hypoxyprobe-1 (in association with reduced oxygenation), and these cells can be easily detected using immunohistochemical techniques employing a commercially available monoclonal antibody that recognizes hypoxyprobe-1-protein adducts (Shabsigh et al, 2001). Tissues from these rats (bladder and kidney) were removed, fixed, and embedded, and sections were then immunostained for hypoxyprobe-1 adducts. Microscopic analysis of kidney sections from all groups (controls and PBOO) demonstrated the typical renal regions of reactivity with the hypoxyprobe antibody (not shown here), and these were considered to be a positive control of antibody staining for each individual rat. Bladder sections from control (unoperated or sham-operated) hypoxyprobe-1-treated rats were uniformly unreactive with the antibody (Fig. 1), thus demonstrating that the urinary bladder under normal conditions shows no areas of cellular hypoxia. In contrast, bladders ob- tained from rats at 3 days post-PBOO contained cells that were found to stain intensely for hyproxyprobe-1 adducts almost uniformly within the mucosal and submucosal regions of the tissue (Fig. 1). However, at 1 week post-PBOO, there no longer was staining of these mucosal-submucosal regions, but extensive hypoxyprobe- 1 antibody binding was now detected in the fibroblasts throughout the outer muscularis and serosa of the bladder (Fig. 1). At 2 weeks post-PBOO, the hypoxyprobe- 1 staining pattern of the bladder had changed again. At this time, focal regions of the bladder muscularis (especially in smooth muscle cells) showed strong reactivity typical of hypoxia (Fig. 1, D and $\mathrm{E})$. These results confirm that the rat bladder does have focal regions of hypoxia after PBOO, but the distinct changes in the hypoxic regions over time suggests that the bladder experiences profound and continual changes in blood flow in response to this hypoxia that leave other regions of the bladder with less than adequate blood flow.

\section{Induction of HIF-1 $\alpha$ in Rat Bladders Subsequent to PBOO}

Individual bladders were recovered from rats, in groups treated similarly to those above $(n=2)$, and the tissues were flash frozen for subsequent protein extraction. Individual bladder extracts containing equal aliquots of protein were electrophoresed on SDS-PAGE gels and the gels were transferred to a nitrocellulose filter to make a Western blot. The Western blot membrane was first probed with an antibody against the human HIF-1 $\alpha$ protein. This antibody recognized a $120 \mathrm{kd}$ protein that was not detected in control (nonobstructed bladders extracts), but was highly induced in all obstrücted bladders (Fig. 2). The blot was then cleaned and reprobed with an antibody that detects the smooth muscle actin protein. This 43 $\mathrm{kd}$ protein was found to be expressed constitutively in control rat bladders and its expression was unchanged over the 2-week period of obstruction. These results were reproduced using individual extracts from the second set of rat bladders.

\section{Induction of an Angiogenic Response in Obstructed Rat Bladders}

Similar Western blots were probed for the expression of the proangiogenic proteins, VEGF and angiopoietin-1. Expression of VEGF mRNA is upregulated by the HIF-1 transcription complex, thus evidence for VEGF up-regulation in the bladder following outlet obstruction could indicate the functionality of the hypoxia-response in the bladder. As shown in Figure 2, both of these proteins were also up-regulated in individual bladders following PBOO in a manner that suggests that the bladder mounts an effective angiogenic response to the hypoxia. To evaluate whether PBOO might also affect the expression of some antiangiogenic regulators, we also probed these blots for the expression of angiopoietin-2 and endostatin. Angiopoietin-2 expression was not detected in any of the bladder extracts (control or 



Figure 1.

Immunostaining profile for hypoxyprobe-1 adducts in bladders of control or partially obstructed rats. Bladders removed from sham-operated rats $(A, \times 40$ magnification or; $\mathrm{B}, \times 200)$; or after 3 days $(\mathrm{C}, \times 40$ or; $\mathrm{D}, \times 200)$; 7 days $(\mathrm{E}, \times 40$ or; $\mathrm{F}, \times 200)$; or 14 days $(\mathrm{G}, \times 40$ or; $\mathrm{H}, \times 200)$ subsequent to partial bladder outlet obstruction (PBOO) were fixed, embedded, and sectioned for immunostaining. Sections were immunostained (brown) with an antibody that recognizes hypoxyprobe-1 adducts, which are formed in hypoxic cells. Sections were subsequently stained with hematoxylin. Results show extensive reactivity with hypoxyprobe- 1 antibody in cells of the mucosa/submucosa (C, D) or in fibroblasts throughout the outer muscularis and serosa (E, F), progressing to groups of smooth muscle cells within the muscularis $(G, H)$. A, Panel marked with arrows to identify mucosa $(M)$ or serosa $(S)$ region of the bladder.

obstructed) even though the antibody suitably recognized a prominent $62 \mathrm{kd}$ band present in extracts of the prostate cancer cell line, LNCaP (data not shown). Enigmatically, Western blot analysis of the obstructed bladder extracts demonstrated the progressive up-regulation of endostatin, a potent antiangiogenic molecule, following PBOO. Endostatin protein levels were 9-fold higher in the 2-week 

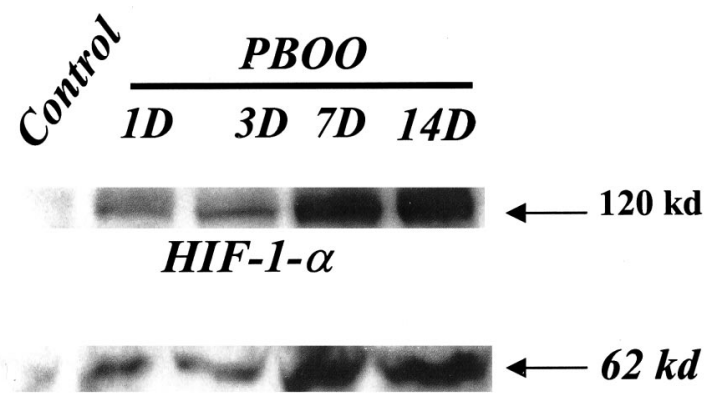

Angiopoietin 1


Figure 2.

Western blot analysis demonstrates that PBO0 activates the hypoxia response and expression of pro and antiangiogenic molecules in the rat bladder. A Western blot containing equal amounts of protein from extracts of control (nonobstructed rat bladder tissue) or obstructed bladder tissues (at various times, as indicated). First panel, expression of hypoxia inducible factor- $1 \alpha$ (HIF-1 $\alpha$ ); second panel, expression of angiopoietin-1; third panel, expression of vascular endothelial growth factor (VEGF); fourth panel, expression of endostatin; fifth panel, expression of $\alpha$-smooth muscle actin (control gene product). Results show that PBO0 activates the bladder's hypoxia response and stimulates expression of pro (VEGF and angiopoietin-1) and antiangiogenesis (endostatin) molecules.

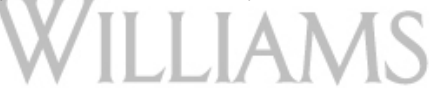

obstructed rats when compared with control (unoperated) rats.

\section{Discussion}

To better understand the effects of bladder outlet obstruction on bladder structure and function, several animal models have been developed and used for research purposes, including rats, rabbits, guinea pigs, cats, dogs, and pigs (Kato et al, 1988; Levin et al, 1990a; Radzinski et al, 1991; Rohner et al, 1978; Seki et al, 1992; Speakman et al, 1991; Steers and DeGroat, 1988). Rats and rabbits subject to experimental partial bladder obstruction eventually develop pathologic changes in their bladders that closely mimic the pathologic changes found in the bladders of humans with benign prostatic hyperplasia (Levin et al, 1990b, 2000). Previous studies of these animal models have shown that PBOO initially enlarges the bladder mass, and this is accompanied by smooth muscle hypertrophy and hyperplasia of fibroblasts and the urothelium. These hypertrophic changes allow the bladder to temporarily counteract the resistance of the obstruction. Eventually, however, bladder detrusor function deteriorates and the obstructed bladder undergoes decompensation, a process associated with the loss of smooth muscle function and increased collagen deposition (Kato et al, 1990; Kim et al, 2000; Levin et al, 1990b; Saito et al, 1993). Increased collagen deposition results in a progressive decrease of compliance, which, in turn, is correlated with further progression of decompensation (Levin et al, 1992).

There are several reasons to suspect that alterations in bladder blood flow (associated with the onset of ischemia/hypoxia) play a role in this progressive process. For one, the placement of the outlet obstruction rapidly leads to bladder overdistension, as the normal bladder is unable to properly empty when faced with the obstruction (Shabsigh et al, 2000). Prior studies of bladder blood flow during various states of distention have shown that blood flow rapidly diminishes as the bladder becomes overdistended (Lieb et al, 2000). Likewise, prior experimental work comparing the early gene expression patterns of partially obstructed rabbit bladders with partially ischemic rabbit bladders identified distinct similarities in the gene products that were induced by these two very dissimilar stresses, suggesting that they share a common etiology (Chen et al, 1996). Finally, direct studies of bladder blood flow in partially obstructed rabbit bladders have correlated the loss of bladder function in this model with an abnormally low rate of blood flow to the bladder muscularis (Levin et al, 1990b; Rohner et al, 1978). Here, we have studied biomarkers of cellular hypoxia (formation of hypoxyprobe-1 adducts and expression of HIF-1 $\alpha$ protein), and our results confirm the extensive occurrence of cellular hypoxia in association with PBOO in the rat bladder model system.

In the current study, the hypoxyprobe-1 labeling technique allowed us to directly identify hypoxic cells in the partially obstructed rat bladder. Our results show that control (unoperated and sham-operated) rat bladders do not contain hypoxic cells. Interestingly, there were no hypoxic areas over the first 24 hours demonstrating clearly that the surgical placement of the ligature does not itself induce ischemia. However, within 3 days of PBOO, cells within the mucosa/ submucosa become intensely reactive with hypoxyprobe-1 demonstrating that this limited region of the bladder is now hypoxic. One week after PBOO, the bladder mucosa/submucosa no longer demonstrates reactivity with hypoxyprobe-1, suggesting that over this time interval ( 3 days to 1 week post-PBOO) it is has established an increased circulation to this region. Indeed, this is consistent with the recent report of Levin and colleagues who used immunohistochemical techniques to identify and quantify vascular components in obstructed rabbit bladders and demonstrated extensive neovascular development within the bladders' submucosal region at a similar early time subsequent to PBOO (Chichester et al, 2000, 2001). After this (1 week post-PBOO), however, fibroblasts within the outer bladder layer (serosa) as well as those interspersed within the smooth muscle layer were found to be intensely hypoxyprobe-1 reactive, whereas the bladder urothelium was unreactive. This suggests that the newly increased circulation to the inner mucosal/submucosal region of the bladder early 
after PBOO leads to deficits in blood flow to the outer layer of the bladder. It is of even further interest that the serosal layer of the obstructed bladder was found to be the most prominent secondary site of neovascularization, initiating nearly the same time that we detected hypoxia within this region (Chichester et al, $2000,2001)$. These coincidental results (early hypoxia followed by neovascularization of the mucosa/submucosa and later hypoxia followed by neovascularization of the serosa) suggest that hypoxia in any specific regions of the bladder is a potent stimulus for neovascularization. This is consistent with our current understanding that a typical cellular hypoxia response (mediated by the up-regulation of $\mathrm{HIF}-1 \alpha$ ) induces the expression of proangiogenic gene products such as VEGF. Indeed, our analysis of HIF-1 $\alpha$ protein expression demonstrated that this transcriptional activator was highly up-regulated in the post-PBOO rat bladder and that its induction was coincidentally accompanied by the induction of both VEGF and angiopoietin-1 expression in the obstructed bladder.

In the most chronically obstructed rat bladders (2 weeks post-PBOO), we found that hypoxia had finally established within the bladder smooth muscle cells themselves. Levin's analysis of neovascularization during post-PBOO bladder development never found evidence that vascularity was increased within the smooth muscle layer of the bladder, even after chronic obstruction (Chichester et al, 2001). Thus, for some reason, unlike the mucosa/submucosa and serosa layers, once hypoxia occurs in the smooth muscle layer of the obstructed bladder, it is unable to stimulate a regional angiogenic response. Based on our analysis of endostatin expression (an antiangiogenic molecule) in the chronically obstructed rat bladder, we propose that the intense accumulation of this substance during the later stages of PBOO might be a factor associated with the inability of the hypoxic smooth muscle layer to attract the development of new blood vessels. If so, chronic hypoxia of the smooth muscle layer of the partially obstructed bladder is certain to be an important aspect in the eventual loss of bladder smooth muscle function. It is of further interest to note that endostatin is not a primary gene product, rather a $20 \mathrm{kd} \mathrm{C-terminal} \mathrm{degradation} \mathrm{frag-}$ ment of collagen XVIII. Thus, the buildup of collagen associated with PBOO, previously thought to contribute to bladder contractile dysfunction by stiffening the bladder, might also contribute to the development of this dysfunction by suppressing the ability of the hypoxic smooth muscle cells to recover from hypoxia.

In summary, these studies confirm that partial bladder outlet obstruction in the rat induces regional hypoxia in the bladder that changes over time and a subsequent angiogenic response characterized by increased expression of proangiogenic substances. Our results support the concept that hypoxia and HIF- $1 \alpha$ action are positive mediators of acute bladder remodeling subsequent to $\mathrm{PBOO}$. However, the failure of the smooth muscle layer of the bladder to acquire a neovasculature and recover from hypoxia during chronic PBOO is likely related to the eventual development of bladder contractile function.

\section{Material and Methods}

\section{Laboratory Animals}

Mature male Sprague-Dawley rats (325-350 gms) purchased from Camm, Inc. (Camden, New Jersey) were maintained in a controlled environment with food and water available ad libitum under 12 hour light/dark cycles. Upon arrival, animals were randomly assigned to experimental groups. In experiment one, we studied four groups of rats $(n=4)$. One group was shamoperated and maintained for 3 days, whereas the three other groups received PBOO and were subsequently maintained for 3,7 , or 14 days before killing. All rats in these groups were injected 2 hours before euthanasia with a $60 \mathrm{mg} / \mathrm{kg}$ intraperitoneal solution of Hypoxyprobe-1 (pimonidazole; Natural Pharmacia International, Inc., Research Triangle Park, North Carolina) in saline (Shabsigh et al, 2001). Bladders and the left kidneys were then recovered from these rats under anesthesia and the tissues were immediately immersed in $10 \%$ phosphate buffered formalin for 8 hours. The rats were then killed. The fixed bladders and kidneys were routinely processed by dehydration and embedding in paraffin for sectioning and immunostaining. Bladders were mounted in the paraffin blocks so as to obtain full thickness sections that included the base and the dome.

In a second experiment we evaluated four different groups $(n=4)$ that received partial bladder outlet obstructions under anesthesia by means of a surgical procedures previously described (Shabsigh et al, 2000) and were then euthanized at 3 days, or 1,2 , or 4 weeks after $\mathrm{PBOO}$ to obtain tissue for subsequent protein extraction and analysis for HIF- $1 \alpha$, endostatin, and angiogenic factors production.

\section{Immunohistochemistry for Hypoxyprobe-I Adducts in Bladders of Obstructed Rats}

Serial $5 \mu \mathrm{M}$ full-thickness cross sections of bladder (from the dome to base) and kidney were cut from paraffin blocks with a microtome and the sections were attached to positive-charged slides. The sections were deparaffinized in xylene and then rehydrated through graded alcohols. Sections were treated with Protease I solution (Ventana Medical Systems, Tucson, Arizona) for antigen retrieval and were then stained with a mouse monoclonal antibody (Mab-I, 1:50 dilution; Natural Pharmacia International, Inc.) on the Ventana ES automated immunostainer (Ventana Medical Systems), using conditions described by the antibody supplier. Antibody binding was detected following incubation with secondary biotinylated goat antimouse (Lab Vision, Fremont, California) and reagents in the Vectastain immunohistochemical staining kit (Vector Laboratories, Inc., Burlingame, California). Immunostained sections were lightly counterstained with hematoxylin and were then dehydrated and mounted for observation. We evaluated eight different sections from each bladder or kidney. 


\section{Protein Extraction and Western Blotting for HIF-1a, VEGF-A, Angiopoietin-1, or Endostatin}

Frozen tissues were pulverized under liquid nitrogen and the frozen powder was then thawed and homogenized (using a Polytron tissue homogenizer; Brinkmann Instruments, Inc., Westbury, New York) in 5 volumes of SDS buffer (120 mm Tris-HCL, pH 6.8, 4\% SDS). The homogenate was stored on ice for 40 minutes before the removal of nuclear and other debris by centrifugation at $14000 \times g$ for 15 minutes. Protein concentrations in the extracts were determined using the Bio-Rad Protein Assay system (BioRad Labs, Inc., Richmond, California). Aliquots of extracts containing $50 \mu \mathrm{gs}$ of protein were solubilized in loading buffer (10\% glycerol, 2\% SDS, 60 mM Tris, $\mathrm{pH}$ 6.8, 0.01\% bromophenol blue, and 100 mм DTT) and were boiled for 5 minutes before electrophoresis on $12 \%$ SDS-polyacrylamide gels. The proteins in the gel were electrophoretically transferred to nitrocellulose membranes (Hybond; Amersham, Inc., Arlington Heights, Illinois) to produce Western blots in $25 \mathrm{~mm}$ Tris-HCL, pH 8.0, 192 mm Glycine, and 20\% methanol at 100 volt for 1 hour. Membranes were blocked in $10 \%$ nonfat milk powder (in TBS-T, 20 mM Tris-HCL, $\mathrm{pH} 7.6,136 \mathrm{~mm} \mathrm{NaCl}, 0.3 \%$ Tween-20) at $4^{\circ} \mathrm{C}$ overnight and were then incubated for 1 hour with a mouse monoclonal antibody against human HIF-1 $\alpha$ (diluted 1:1000; Novus Biologicals, Littleton, Colorado), or with mouse monoclonal antibody against rat VEGF (Santa Cruz Biotechnology, Inc., Santa Cruz, California) diluted 1:100 in TBS-T buffer at $37^{\circ} \mathrm{C}$ (for detection of VEGF), or with goat polyclonal antibodies against rat Angiopoietin-1 (diluted 1:200, Santa Cruz Biotechnology, Inc.) or goat polyclonal antibody against rat endostatin (diluted 1:500; a gift from Dr. Jonathan Barach). After extensive washing with TBS-T buffer, membranes were incubated with a secondary antibody (sheep antimouse IgG-horseradish peroxidase complex for VEGF and HIF- $1 \alpha$ or goat anti-goat IgG-horseradish peroxidase for angiopoietin-1 or endostatin; Santa Cruz Biotechnology, Inc.). Chemoluminescent detection of antibody binding was performed using the ECL Western blotting analysis system (Amersham, Inc.). Subsequently all Western blots were cleaned by incubation in a denaturing buffer $\left(0.2 \%\right.$ SDS, $0.1 \mathrm{~mm}$ DTT) at $70^{\circ}$ for 30 minutes followed by several washings in TBS-T and reblocking with $10 \%$ milk solution. The blot was then reprobed for smooth muscle actin using (mouse monoclonal diluted 1:2000; Sigma Chemical Company, Inc., St Louis, Missouri). The autoradiograph was analyzed with the Molecular Dynamics Scanning Densitometer (Sunnyvale, California).

\section{References}

Arteel GE, Thurman RG, and Raleigh JA (1998). Reductive metabolism of the hypoxia marker pimonidazole is regulated by oxygen tension independent of the pyridine nucleotide redox state. Eur J Biochem 253:743-750.

Blaivas JG (1996). Obstructive uropathy in the male. Urol Clin North Am 23:373-384.
Buttyan R, Jacobs BZ, Blaivas J, and Levin RM (1992). The early molecular response to rabbit bladder outlet obstruction. Neurourol Urodyn 11:225-238.

Chen MW, Buttyan R, and Levin RM (1996). Genetic and cellular response to unilateral ischemia of the rabbit urinary bladder. J Urol 155: 732-737.

Chichester P, Lieb J, Levin SS, Buttyan R, Horan P, and Levin RM (2000). Vascular response of the rabbit bladder to short term partial outlet obstruction. Mol Cell Biochem 208:19-26.

Chichester P, Schroder A, Horan P, and Levin RM (2001). Vascular response of the rabbit bladder to chronic partial outlet obstruction. Mol Cell Biochem 226:1-8.

Kato K, Wein AJ, Kitada S, Haugaard N, and Levin RM (1988). The functional effect of mild outlet obstruction on the rabbit urinary bladder. J Urol 140:880-884.

Kato K, Wein AJ, Longhurst PA, Haugaard N, and Levin RM (1990). Effects of outlet obstruction on glucose metabolism of the rabbit urinary bladder. J Urol 143:600-606.

Kim JC, Yoon JY, Seo SI, Hwang TK, and Park YH (2000). Effects of partial bladder outlet obstruction and its relief on types I and III collagen and detrusor contractility in the rat. Neurourol Urodyn 19:29-42.

Levin RM, Brading AF, Mills IW, and Longhust PA (1999). Prostatic disease. St. Louis: WB Saunders Co.

Levin RM, Haugaard N, O'Connor L, Buttyan R, Das A, Dixon JS, and Gosling JA (2000). Obstructive response of human bladder to BPH vs. rabbit bladder response to partial outlet obstruction: A direct comparison. Neurourol Urodyn 19:609629 .

Levin BM, Longhurst PA, Barasha B, McGuire EJ, Elbadawi A, and Wein AJ (1992). Studies on experimental bladder outlet obstruction in the cat: Long-term functional effects. JUrol 148:939-943.

Levin RM, Longhurst PA, Kato K, McGuire EJ, Elbadawi A, and Wein AJ (1990a). Comparative physiology and pharmacology of the cat and rabbit urinary bladder. J Urol 143:848852.

Levin RM, Longhurst PA, Monson FC, Kato K, and Wein AJ (1990b). Effect of bladder outlet obstruction on the morphology, physiology, and pharmacology of the bladder. Prostate 3(Suppl):9-26.

Levin RM, Memberg W, Ruggieri MR, and Wein AJ (1986). Functional effects of in vitro obstruction on the rabbit urinary bladder. J Urol 135:847-851.

Lieb JI, Chichester P, Kogan B, Das AK, Leggett RE, Schroder A, and Levin RM (2000). Rabbit urinary bladder blood flow changes during the initial stage of partial outlet obstruction. J Urol 164:1390-1397.

Radzinski C, McGuire EJ, Smith D, Wein AJ, Levin RM, Miller LF, and Elbadawi A (1991). Creation of a feline model of obstructive uropathy. J Urol 145:859-863.

Rohner RJ Jr, Hannigan JD, and Sanford EJ (1978). Altered in vitro adrenergic responses of dog detrusor muscle after chronic bladder outlet obstruction. Urology 11:357-361.

Saito M, Wein AJ, and Levin RM (1993). Effect of partial outlet obstruction on contractility: Comparison between severe and mild obstruction. Neurourol Urodyn 12:573-583. 
Seki N, Karim OM, and Mostwin JL (1992). Changes in electrical properties of guinea pig smooth muscle membrane by experimental bladder outflow obstruction. Am J Physiol 262:885-891.

Shabsigh A, Ghafar MA, de la Taille A, Burchardt M, Kaplan SA, Anastasiadis AG, and Buttyan R (2001). Biomarker analysis demonstrates a hypoxic environment in the castrated rat ventral prostate gland. J Cell Biochem 81:437-444.

Shabsigh A, Hayek OR, Weiner D, Saidi J, Kaplan SA, Kiss A, Burchardt M, Buttyan R, and Levin RM (2000). Acute increase in blood flow to the rat bladder subsequent to partial bladder outlet obstruction. Neurourol Urodyn 19:195-206.

Speakman MJ, Brading AF, Dixon JS, Gilpin SA, Gilpin CJ, and Gosling JA (1991). Cystometric, physiological and morphological studies after relief of bladder outflow obstruction in the pig. J Urol 68:243-247.
Steers WD and DeGroat WC (1988). Effect of bladder outlet obstruction on micturition reflex pathways in the rat. J Urol 140:864-871.

Wang GL, Jiang B-H, Rue EA, and Semenza GL (1995). Hypoxia-inducible factor 1 is a basic-helix-loop-helix-PAS heterodimer regulated by cellular $\mathrm{O} 2$ tension. Proc Natl Acad Sci USA 92:5510-5514.

Wein AJ (1995). Bladder outlet obstruction-An overview. Adv Exp Med Biol 385:3-5.

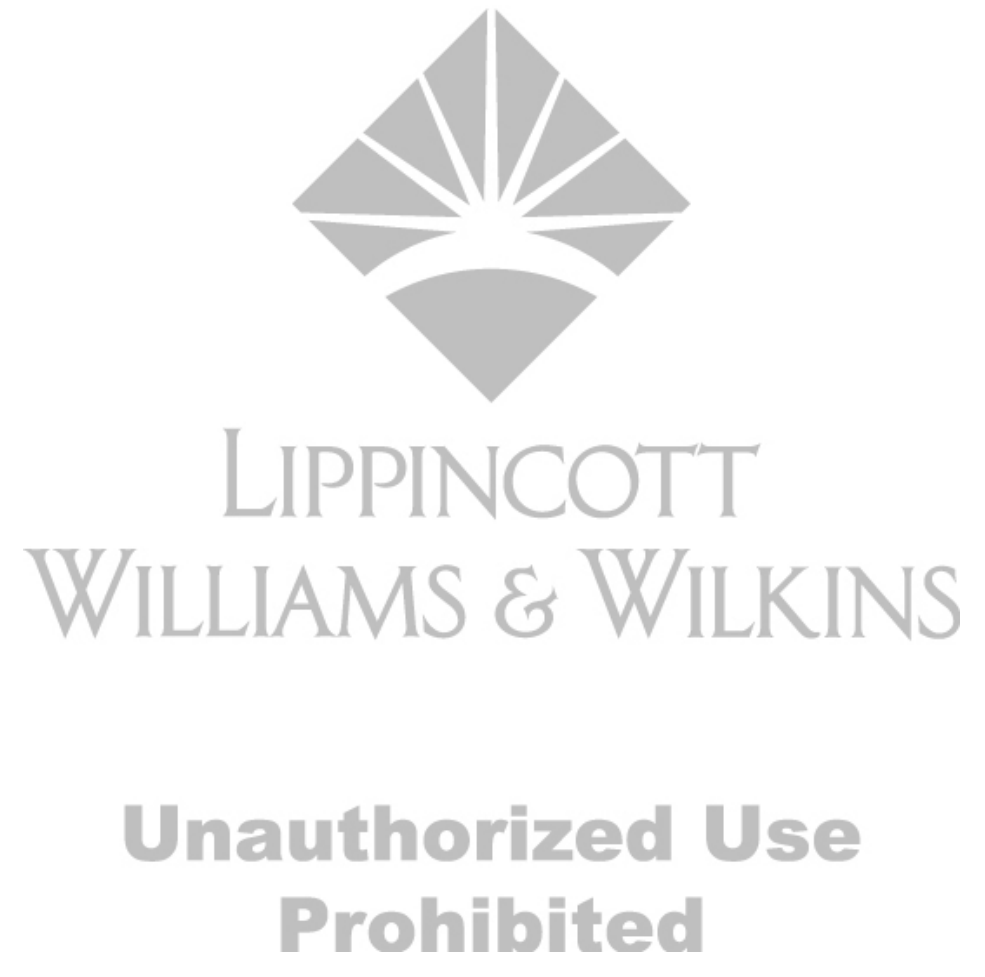

